\title{
Mejora de la Competencia Digital del futuro profesorado de Educación Secundaria: Enseñanza y Aprendizaje con TIC
}

\section{Improvement of the Digital Competence of future Secondary Education teachers: Teaching and Learning with ICT}

ANTONIO PALACIOS-RODRIGUEZ

ORCID: https://orcid.org/0000-0002-0689-6317

Universidad de Sevilla

Didáctica y Organización Educativa

aprodriguez@us.es

Fecha de recepción: 18/11/2019

Fecha de aceptación: 21/11/2019

DOI: $h$ ttp://dx.doi.org/10.12795/9788447221912.099

Pp.: 2223-2241 


\section{Resumen}

Este trabajo presenta la aplicación de un Ciclo de Mejora Docente en la asignatura "Enseñanza y aprendizaje con TIC", dentro del Master Universitario en Profesorado de Enseñanza Secundaria Obligatoria y Bachillerato, Formación Profesional y Enseñanza de Idiomas (MAES). En él, se trabajan conceptos relacionados con la competencia digital del docente de Educación Secundaria: compromiso profesional y recursos digitales. Los resultados demuestran que el Ciclo de Mejora Docente, basado en un modelo de aprendizaje a partir de la investigación, sirve para aumentar el nivel de competencia digital del alumnado. Además, se evidencia el alto grado de implicación.

Palabras claves: Enseñanza y Aprendizaje con TIC, MAES, Docencia Universitaria, Experimentación Docente Universitaria, Competencia Digital.

\section{Abstract}

This paper presents the application of a Teaching Improvement Cycle in the subject "Teaching and learning with ICT", University Master's Degree in Teaching of Secondary and Secondary Education, Vocational Training and Language Teaching. In it, concepts related to the digital competence of the Secondary Education teacher are worked on: professional engagement and digital resources. The results show that Teaching Improvement Cycle, based on a researching learning model, increases the level of students digital competence. In addition, the high degree of involvement is evident.

Keywords: Teaching and Learning with ICT, University Master's Degree in Teaching of Secondary and Secondary Education, Vocational Training and Language Teaching, University Teaching, University Teaching Experimentation, Digital Competence.

Jornadas de Formación e Innovación Docente del Profesorado | № 2 (2019) Esta obra se distribuye con la licencia Creative Commons Reconocimiento-NoComercial-SinObraDerivada Internacional (CC BY-NC-ND 4.0.) 


\section{Introducción}

Este ciclo de mejora docente ha sido realizado en la asignatura "Enseñanza y Aprendizaje con TIC" del Master Universitario en Profesorado de Enseñanza Secundaria Obligatoria y Bachillerato, Formación Profesional y Enseñanza de Idiomas (MAES). Además, surge de las demandas directas del alumnado, canalizadas a través de un cuestionario inicial elaborado con Google Forms denominado "datos de contacto y otras cuestiones" en el que se plantea, entre otras cuestiones, "qué espera de la asignatura", "sobre qué TIC le gustaría profundizar" y "cómo le gustaría que fueran las clases". La Tabla 1 recoge alguna de las respuestas. En todo momento se cuenta con el consentimiento del alumnado para publicar sus respuestas anonimizadas.

Tabla 1. Respuestas obtenidas por el alumnado en el cuestionario "datos de contacto y otras cuestiones"

\begin{tabular}{|c|c|c|}
\hline $\begin{array}{l}\text { ¿Qué esperas de } \\
\text { esta asignatura? (a } \\
\text { parte de aprobarla). }\end{array}$ & $\begin{array}{c}\text { ¿Qué nuevas tecnologias } \\
\text { te gustaría descubrir en la } \\
\text { asignatura? }\end{array}$ & $\begin{array}{c}\text { ¿Cómo puedo ayudarte } \\
\text { este curso? }\end{array}$ \\
\hline $\begin{array}{l}\text { Aprender los } \\
\text { programas que se } \\
\text { utilizan actualmente en } \\
\text { las aulas. }\end{array}$ & Programas de enseñanza. & $\begin{array}{l}\text { A manejar programas } \\
\text { que se utilizan en las } \\
\text { aulas para hacerlas más } \\
\text { dinámicas. }\end{array}$ \\
\hline $\begin{array}{l}\text { Aprender sobre } \\
\text { recursos que utilizar en } \\
\text { el aula en un futuro }\end{array}$ & $\begin{array}{l}\text { Relacionadas con la } \\
\text { enseñanza de música (mi } \\
\text { especialidad) }\end{array}$ & $\begin{array}{l}\text { Enseñándome } \\
\text { herramientas, ideas, } \\
\text { aplicaciones, etc. sobre } \\
\text { cómo utilizar las TIC } \\
\text { de forma interesante y } \\
\text { significativa }\end{array}$ \\
\hline $\begin{array}{l}\text { Aprender alternativas y } \\
\text { fórmulas de transmitir } \\
\text { conocimientos }\end{array}$ & $\begin{array}{l}\text { Estoy abierto al aprendizaje } \\
\text { de cualquiera }\end{array}$ & Con ejemplos prácticos \\
\hline $\begin{array}{l}\text { Aprender a manejar } \\
\text { medios audiovisuales } \\
\text { para incoporar en mi } \\
\text { docencia }\end{array}$ & $\begin{array}{l}\text { Software libre. Sólo conozco } \\
\text { kahoot y power point (que } \\
\text { no es gratuito), por lo tanto } \\
\text { cualquier ampliación de esto } \\
\text { lo considero positivo }\end{array}$ & $\begin{array}{l}\text { Enseñándome a manejar } \\
\text { recursos tecnológicos que } \\
\text { pueda incorporar en mi } \\
\text { docencia para dinamizar } \\
\text { el aula }\end{array}$ \\
\hline
\end{tabular}

Jornadas de Formación e Innovación Docente del Profesorado | № 2 (2019) Esta obra se distribuye con la licencia Creative Commons Reconocimiento-NoComercial-SinObraDerivada Internacional (CC BY-NC-ND 4.0.) 
Como se puede observar, la mayoría hace referencia al conocimiento y uso de recursos TIC para su posterior integración en el aula. Además, también se menciona el aprendizaje significativo, flexible y centrado en el alumnado. Por este motivo, el ciclo de mejora se centra en el desarrollo de una investigación sobre recursos TIC, formación docente relacionada y posterior integración en el aula de secundaria.

\section{Contexto}

Como se ha comentado en el apartado anterior, este ciclo de mejora se desarrolla en la asignatura "Enseñanza y Aprendizaje con TIC", dentro del Master Universitario en Profesorado de Enseñanza Secundaria Obligatoria y Bachillerato, Formación Profesional y Enseñanza de Idiomas (MAES) de la Universidad de Sevilla. Esta asignatura, de 2 créditos, es impartida durante el 1er cuatrimestre y tiene carácter optativo. Además, es compartida entre dos departamentos de la Universidad de Sevilla: Ingeniería Gráfica (Escuela Técnica Superior de Ingeniería de Edificación) y Didáctica y Organización Educativa (Facultad de Ciencias de la Educación). Existen un total de 4 grupos, 2 por departamento, con 4 profesores distintos. El nivel de coordinación entre los docentes es bueno, sobre todo entre los del mismo departamento, lo que ha facilitado el desarrollo del ciclo de mejora.

En relación al alumnado, se cuenta con 27 estudiantes matriculados: 14 alumnos y 13 alumnas. La media de edad es 28 años. La mayoría provienen de Sevilla capital (10) y pueblos de la provincia (10). Existe una gran variedad de titulaciones de origen (Figura 1). Todos afirman que la elección del máster era su primera opción. Además, ningún estudiante manifiesta tener alguna necesidad específica de aprendizaje. 


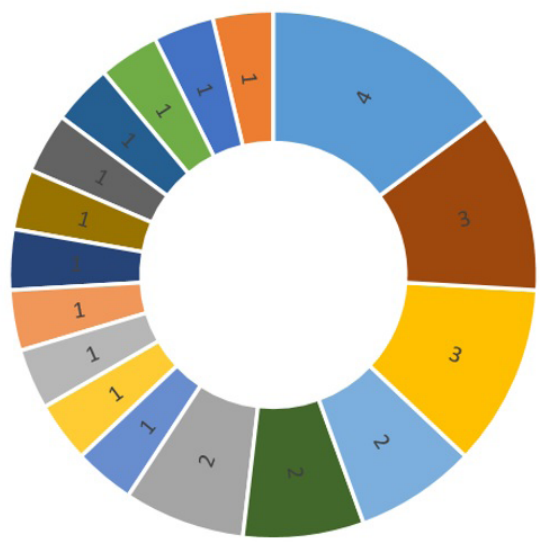

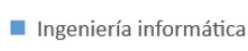

arquitectura

- Biología

- Economía

- Ingeniería telecom.

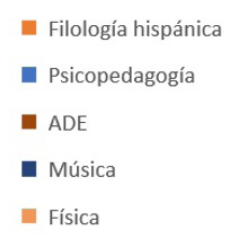

- Relaciones internacionales auímica

- Educación Primaria

- Matemáticas

- Ingeniería industrial

Educación social

Figura 1. Número de estudiantes por titulación de origen

\section{Objetivos}

El principal objetivo de este ciclo de mejora es aumentar el nivel de competencia digital del alumnado a través de 4 sesiones en las que se favorece el aprendizaje basado en la investigación. Concretamente, se pretende:

1. Aumentar el nivel de compromiso profesional con TIC: uso de canales de comunicación digital, trabajo colaborativo con TIC, reflexión activa sobre la competencia digital y formación en línea.

2. Conocer y usar recursos digitales integrables en el aula de educación secundaria: estrategias de búsqueda de recursos digitales, creación y modificación de recursos y protección de contenido digital. 


\section{Ideas iniciales del alumnado}

Analizar los procesos de cambio y los obstáculos que dificultan la progresión en el aprendizaje es una tarea fundamental en una evaluación formativa y para el aprendizaje del alumnado (Rivero y Porlán, 2017). Por este motivo, para explorar las ideas del alumnado, es decir, sus modelos mentales, se parte de un cuestionario inicial anónimo de preguntas abiertas como herramienta capaz de aportar información relevante para adaptar el proceso de enseñanza-aprendizaje. Dicho cuestionario es realizado en la primera sesión y su duración es de aproximadamente 15 minutos. En él se plantean 7 situaciones abiertas (1 por cada contenido) relacionadas con problemas atractivos y relevantes. Se utiliza la herramienta Google Forms por ser capaz de generar cuestionarios de este tipo, dando la posibilidad de descargar las respuestas en forma de matriz de datos (facilidad de análisis). Todo el alumnado contesta a todas las preguntas. Por cada pregunta, se realiza una clasificación de le "respuestas tipo" en forma de "escalera de aprendizaje", atendiendo a su nivel de complejidad. La Figura 2 representa una de estas escaleras. Al ser muy numerosas, es imposible incorporarlas en este documento. sin embargo, el lector puede visualizar esta infografia realizada con la aplicación Genially, donde puede encontrar todas las escaleras en forma de gráfico.

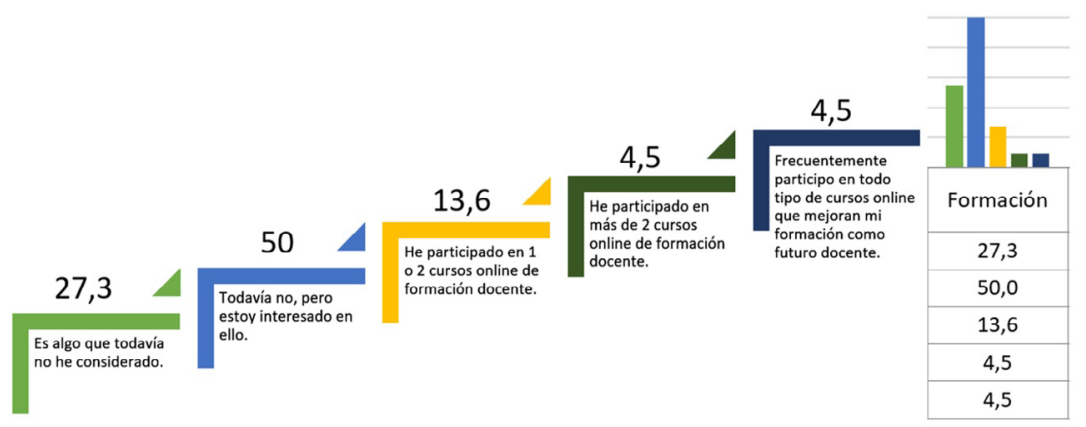

Figura 2. Ejemplo de escalera de aprendizaje: "formación online" preCIMA 
En todos los casos, la mayoría de los alumnos y alumnas se sitúan en el segundo (23,35\%) y tercer nivel (40,25\%). En consecuencia, se diseña una secuencia de actividades para ayudar a superar los obstáculos detectados y ayudarles a avanzar hacia los siguientes niveles.

\section{Contenidos}

El docente debe cuestionarse qué y cómo va a trabajar con el alumnado. En relación al qué trabajar, se elabora un mapa de contenidos basado en los problemas planteados en el cuestionario inicial (Figura 3). Además, dichos contenidos toman como referencia el Marco Europeo de Competencia Digital del Profesorado "DigCompEdu" (Redecker y Punie, 2017), que establece un modelo de desarrollo de competencias digitales alineado con las políticas de la Unión Europea (Consejo de la UE, 2018). Al haberse creado con la aplicación Genially, se recomienda su visualización a través de este enlace.

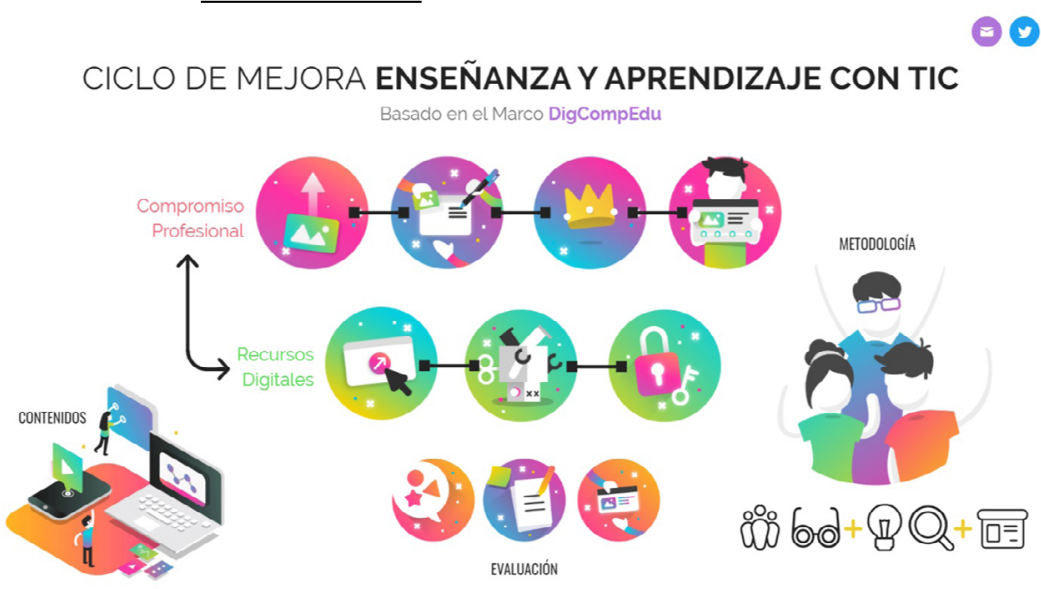

Figura 3. Mapa de contenidos.

En concreto, los contenidos a trabajar en este ciclo de mejora docente son:

Jornadas de Formación e Innovación Docente del Profesorado | № 2 (2019) Esta obra se distribuye con la licencia Creative Commons Reconocimiento-NoComercial-SinObraDerivada 
1. Contenido conceptual
a) Concepto, epistemología e historia de las TIC apli- cadas a la educación .
b) Las tecnologías digitales y la docencia: Educación Secundaria.
c) La integración curricular de los recursos tecnológicos.

2. Contenido procedimental

a) Saber formar grupos heterogéneos.

b) Saber asignar de roles dentro del grupo.

c) Saber crear una carpeta compartida en la nube.

d) Saber crear documentos en la nube.

e) Saber compartir una carpeta en la nube.

f) Saber debatir sobre noticia relacionada con el uso de las TIC en Educación Secundaria.

g) Saber investigar usando fuentes de información fiables.

h) Saber formarse en línea sobre recursos TIC.

i) Saber buscar recursos (infografia, web, blog, wiki, $R A \ldots$...

j) Saber crear recursos (infografia, web, blog, wiki, RA...).

k) Saber modificar recursos (infografia, web, blog, wiki, RA...).

3. Contenido actitudinal

a) Predisposición a desempeñar buenas prácticas con TIC

b) Desarrollo de pensamiento crítico sobre las ventajas e inconvenientes de las TIC en el aula de Educación Secundaria.

\section{Metodología}

La metodología planteada está vinculada a la mejora de la competencia digital del alumnado a través de 
diferentes situaciones que suponen un desafio cognitivo para el mismo. Desde la primera sesión se organizan pequeños grupos, pues suponen: participación del alumnado, aprendizaje cooperativo, interacción y motivación (Finkel, 2008). Al mismo tiempo, el papel del docente es el de supervisar el trabajo de los grupos y soluciona los problemas funcionales y cognitivos que se plantean en cada uno de ellos. En todo momento se intenta conseguir la atención y curiosidad del alumnado, tratando de partir de sus ideas y desarrollando competencias de aprendizaje autónomo mediante propuestas estimulantes y significativas (Bain, 2005). Conjuntamente, se proporciona un espacio para la presentación de resultados finales (exposición final). De esta forma, la experiencia está completa al tener un inicio o presentación del problema, medio o resolución del problema y fin o presentación de resultados. Así, el modelo metodológico que se plantea para todas las sesiones (Figura 4) está basado en:

1. R: Resolución de problemas
a) ¿Qué tipo de recurso digital podemos integrar en el aula de Educación Secundaria?
b) ¿Cómo se usa ese recurso?
c) ¿Cómo se puede integrar?

2. C: Trabajo cooperativo
a) Trabajo grupal
b) Reflexión individual
c) Coevaluación del alumnado

3. T: Uso de las TIC
a) Búsqueda de información en fuentes fiables
b) Investigación sobre temática elegida por el alum- nado, a partir de sus intereses
c) Exposición apoyada en material multimedia

4. F: Sintesis final
a) Exposición oral
b) Entrega de informe

Jornadas de Formación e Innovación Docente del Profesorado | № 2 (2019) Esta obra se distribuye con la licencia Creative Commons 
En todas las fases, el papel del profesor (P) es el de supervisar el trabajo grupal. Éste aporta retroalimentación durante la realización de la tarea, en la entrega del borrador y en la entrega del informe final. Conjuntamente, todo el proceso debe ir ajustándose a las ideas del alumnado (I), de manera que el tema no se impone, el alumnado lo propone. Por este motivo, cada grupo trabaja sobre una problemática relevante para el mismo.

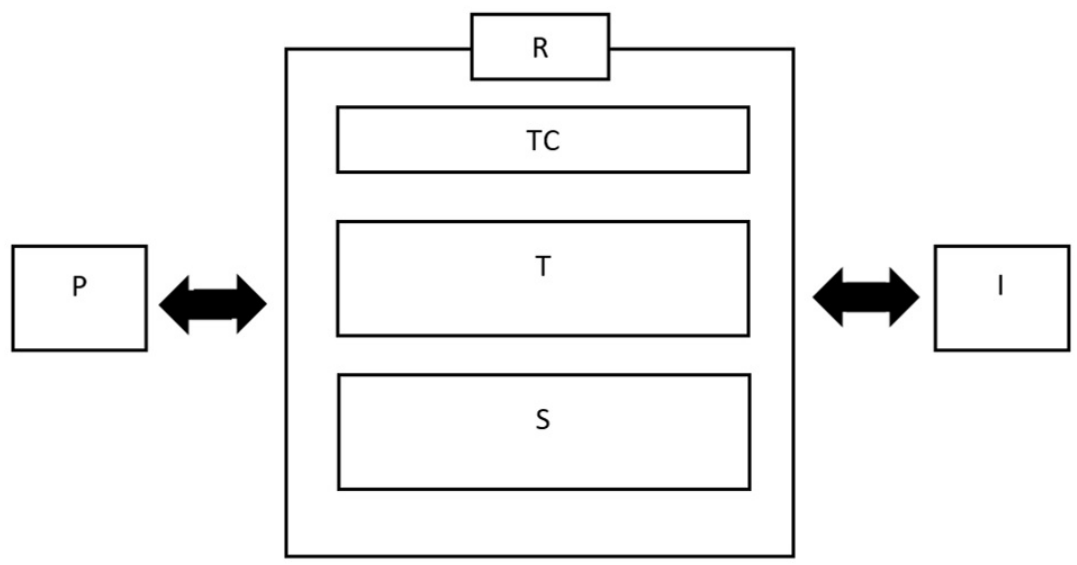

Figura 4. Modelo metodológico.

Jornadas de Formación e Innovación Docente del Profesorado | № 2 (2019) Esta obra se distribuye con la licencia Creative Commons 


\section{Actividades}

A continuación, se describe la secuencia de actividades propuesta (Tabla 2).

Tabla 2. Secuencia de actividades

\begin{tabular}{|c|c|c|c|c|}
\hline Sesión & $\begin{array}{c}\text { Fase del } \\
\text { Modelo } \\
\text { Metodológico }\end{array}$ & Actividad & Descripción & Duración \\
\hline \multirow[t]{2}{*}{1} & $\begin{array}{l}\text { Trabajo } \\
\text { cooperativo. } \\
\text { Uso de las TIC. }\end{array}$ & $\begin{array}{l}\text { A1. Creación } \\
\text { del grupo }\end{array}$ & $\begin{array}{l}\text {-Presentación del alumnado (soy..., puedo } \\
\text { aportar...). } \\
\text {-Creación del grupo (max. } 4 \text { personas). } \\
\text { - Nombre del grupo (consenso). } \\
\text {-Designación de roles. } \\
\text {-Creación de una carpeta One Drive usando } \\
\text { UVUS. } \\
\text {-Subir el enlace de la carpeta a la } \\
\text { plataforma. }\end{array}$ & 1 hora \\
\hline & Uso de las TIC. & $\begin{array}{l}\text { A2. Búsqueda } \\
\text { de noticia }\end{array}$ & $\begin{array}{l}\text { Buscar, como mínimo, una noticia (video o } \\
\text { texto), entrada en blog o debate en foro en } \\
\text { el que se discuta sobre el uso de las TIC en } \\
\text { aulas de Educación Secundaria. } \\
\text { Adjuntar un archivo PDF que contenga la } \\
\text { siguiente información: } \\
\text { - URL donde se encuentra la notica } \\
\text { - Medios tecnológicos identificados en la } \\
\text { noticia } \\
\text { - ¿Crees que está justificado el uso de estos } \\
\text { medios tecnológicos?, ¿por qué? } \\
\text { - ¿Podría(n) haber sido sustituido(s) por } \\
\text { otros recursos?, por ejemplo, ¿cuáles?, ¿qué } \\
\text { ventajas e inconvenientes habría tenido? } \\
\text { - ¿Cuáles son las principales barreras } \\
\text { que puede tener el docente de Educación } \\
\text { Secundaria a la hora de integrar las TIC } \\
\text { en el aula? (justificalo con alguna noticia, } \\
\text { entrevista, artículo científico) }\end{array}$ & 1 hora \\
\hline
\end{tabular}

Jornadas de Formación e Innovación Docente del Profesorado I № 2 (2019)

Esta obra se distribuye con la licencia Creative Commons

Reconocimiento-NoComercial-SinObraDerivada

Internacional (CC BY-NC-ND 4.0.) 


\begin{tabular}{|c|c|c|c|}
\hline Uso de las TIC. & A3. Debate & $\begin{array}{l}\text { Debate sobre las cuestiones planteadas en la } \\
\text { sesión anterior: } \\
\text { - ¿Crees que está justificado el uso de estos } \\
\text { medios tecnológicos?, ¿por qué? } \\
\text { - ¿Podría(n) haber sido sustituido(s) por } \\
\text { otros recursos?, por ejemplo, ¿cuáles?, ¿qué } \\
\text { ventajas e inconvenientes habría tenido? } \\
\text { - ¿Cuáles son las principales barreras } \\
\text { que puede tener el docente de Educación } \\
\text { Secundaria a la hora de integrar las TIC } \\
\text { en el aula? (justificalo con alguna noticia, } \\
\text { entrevista, artículo científico) }\end{array}$ & $1 / 2$ hora \\
\hline Uso de las TIC. & $\begin{array}{l}\text { A4. Resumen } \\
\text { del debate }\end{array}$ & $\begin{array}{l}\text { Reflexionar sobre: } \\
\text { ¿¿Qué has aprendido? } \\
\text {-¿Cuál ha sido tu grado de implicación en el } \\
\text { debate?, ¿ por qué? } \\
\text {-¿Cuál ha sido la parte que más te ha } \\
\text { interesado? } \\
\text {-¿Sobre qué te gustaría profundizar? } \\
\text {-Valoración del debate (ventajas, } \\
\text { inconvenientes, cómo mejorarlo...) }\end{array}$ & $1 / 2$ hora \\
\hline $\begin{array}{l}\text { Resolución de } \\
\text { problemas. } \\
\text { Trabajo } \\
\text { cooperativo. } \\
\text { Uso de las TIC. }\end{array}$ & $\begin{array}{l}\text { A5. } \\
\text { Investigación } \\
\text { (I) }\end{array}$ & $\begin{array}{l}\text { Elegir } 1 \text { medio educativo digital sobre el que } \\
\text { te gustaría investigar (buenas prácticas TIC, } \\
\text { tendencias TIC educación). Buscar evidencias } \\
\text { de ese medio en, al menos: } \\
\text {-2 Prácticas } \\
\text { educativas: noticias, videos, blogs. } \\
\text {-2 artículos de revistas científicas de calidad } \\
\text { (ej: Pixel-Bit, Revista Comunicar...). } \\
\text { Ideas clave del medio según las prácticas } \\
\text { educativas y artículos encontrados (síntesis y } \\
\text { relación de los mismos). }\end{array}$ & $1 / 2$ hora \\
\hline
\end{tabular}




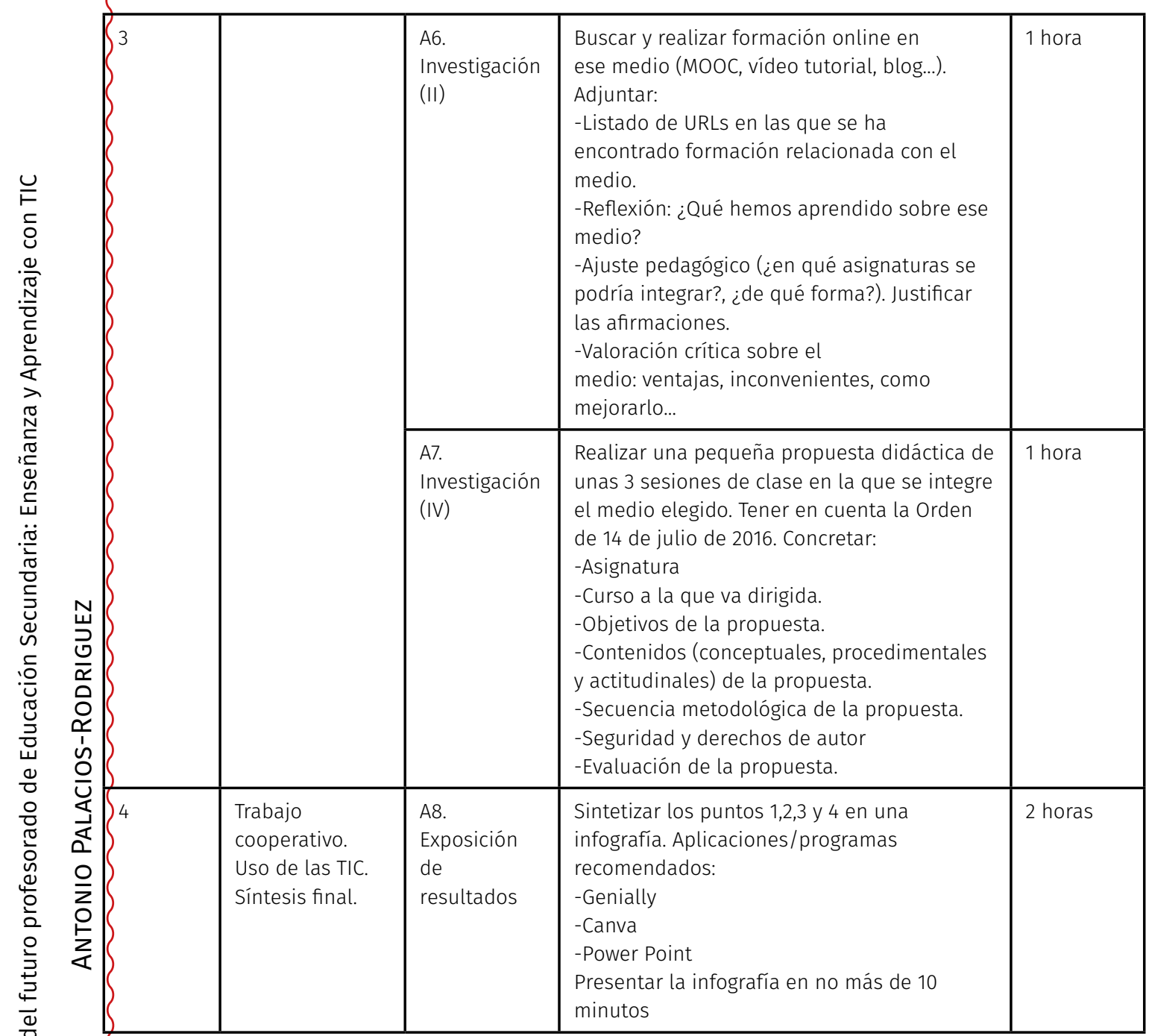

\section{Aplicación del CIMA}

\section{Descripción del desarrollo}

En general, el nivel de satisfacción es alto; no solo del alumnado, que ha dado una retroalimentación positiva 
durante todas las sesiones, sino también del profesor, que ha disfrutado de todas y cada una de ellas. Sin embargo, es cierto que se han producido situaciones de estrés cuando más de un grupo solicitaba la ayuda del profesor al mismo tiempo. Conjuntamente, los tiempos planificados en un primer momento no han sido los reales, teniendo que eliminar parte de algunas actividades. Por ejemplo, la sesión del debate duró más de lo previsto al plantearse como un debate abierto a todo el alumnado. Para próximas sesiones, se planteará un debate en el que primero se comenta por grupos, se elige a un portavoz y éste es el encargado de debatir con el resto de portavoces. Así mismo, se destaca la participación de todo el alumnado: salvo en contadas excepciones, todos los miembros de los grupos han colaborado en todas las tareas que se han ido proponiendo. Se han intentado resolver todos los problemas que iban surgiendo en el grupo. Finalmente, el análisis de las escaleras de aprendizaje (preCIMA y postCIMA) demuestran una evolución positiva. Se puede afirmar que se ha mejorado el nivel de competencia digital a través de un modelo basado en la investigación: conectando con los problemas reales del alumnado, tratando de desarrollar contenidos que van más allá de lo meramente conceptual, abordando la resolución de problemas, evaluando los modelos mentales del alumnado y negociando los ritmos de aprendizaje y evaluación.

\section{Evaluación del aprendizaje de los estudiantes}

La Tabla 3 describe los instrumentos de evaluación utilizados durante el CIMA.

Jornadas de Formación e Innovación Docente del Profesorado | № 2 (2019) Esta obra se distribuye con la licencia Creative Commons 
Tabla 3. Instrumentos de evaluación.

\begin{tabular}{|l|l|l|l|}
\hline \multicolumn{1}{|c|}{ Instrumento } & Quien evalúa & Cuando se usa & Ponderación \\
\hline Cuestionario inicial & Profesor & Inicio del CIMA & $0 \%$ \\
\hline Cuestionario final & Profesor & Final del CIMA & $0 \%$ \\
\hline $\begin{array}{l}\text { Entrega de tareas } \\
\text { individuales } \\
\text { (plataforma) }\end{array}$ & Profesor & A2 y A4 & $20 \%$ \\
\hline $\begin{array}{l}\text { Entrega de } \\
\text { tareas grupales } \\
\text { (plataforma) }\end{array}$ & Profesor & $\begin{array}{l}\text { A1, A3, A5, A6, A7 } \\
\text { y A8 }\end{array}$ & $50 \%$ \\
\hline $\begin{array}{l}\text { Cuestionario } \\
\text { coevaluación } \\
\text { trabajo grupal }\end{array}$ & Alumnado & Final del CIMA & $30 \%$ \\
\hline
\end{tabular}

El aprendizaje se valora a partir del análisis de las escaleras de aprendizaje, elaboradas con los resultados del cuestionario inicial y final. Los resultados del cuestionario preCIMA son mostrados en la Figura 5.

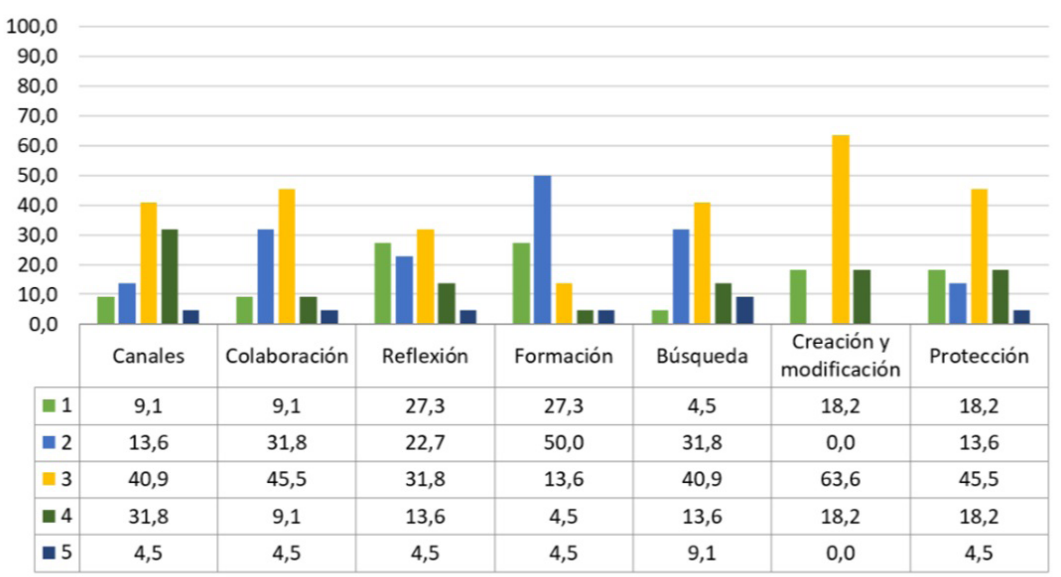

$\square 1=2 \square 3=4 \square 5$

Figura 5. Resultados del cuestionario preCIMA.

Como se puede apreciar, la mayoría de estudiantes se sitúan entre los niveles 2 y 3 , niveles cuyo dominio

Jornadas de Formación e Innovación Docente del Profesorado | № 2 (2019) Esta obra se distribuye con la licencia Creative Commons Reconocimiento-NoComercial-SinObraDerivada Internacional (CC BY-NC-ND 4.0.) 
cognitivo relacionado es recordar (2) y entender (3) cómo se usa la tecnología. Los niveles 4 y 5, cuyos dominios cognitivos están relacionados con la creación (4), integración (5) e innovación (5) con tecnología poseen menor porcentaje de alumnado.

A continuación, se presentan los resultados del cuestionario postCIMA (Figura 6).

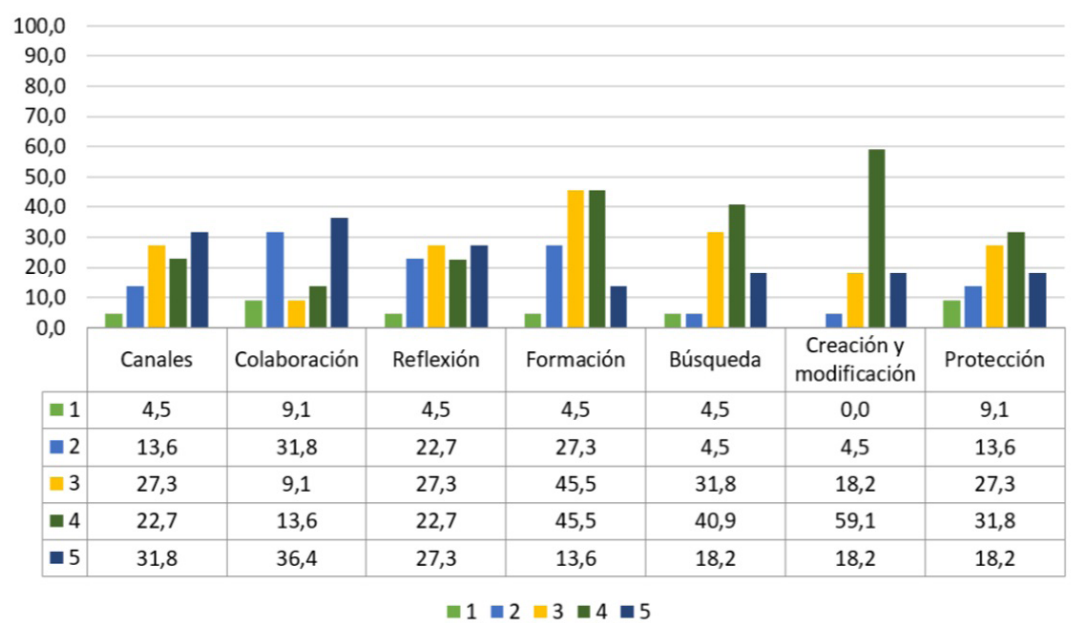

Figura 6. Resultados del cuestionario postCIMA

Tal y como se muestra y, una vez aplicado el CIMA, la mayoría de estudiantes se sitúan entre los niveles 3, 4 y 5, cuyo dominio cognitivo relacionado entender (3), crear (4), integrar (5) e innovar (5) con tecnología. Sin embargo, sigue existiendo un pequeño porcentaje de alumnado situado en los niveles inferiores, aquello relacionados con realizar tareas simples con tecnología (1) y recordar cómo se resuelven problemas básicos con TIC (2).

Finalmente, se realiza un análisis comparativo de los cuestionarios, observable en la Tabla 4. 
Tabla 4. Comparación de resultados del cuestionario preCIMA y postCIMA

\begin{tabular}{|c|c|c|c|c|c|c|}
\hline \multirow{2}{*}{\multicolumn{2}{|c|}{ Nivel }} & \multicolumn{5}{|c|}{ Niveles de progresión } \\
\hline & & 1 & 2 & 3 & 4 & 5 \\
\hline \multirow{7}{*}{$\begin{array}{l}\text { Diferencia } \\
\text { (postCIMA } \\
\text {-preCima) }\end{array}$} & Canales & $-4,5 \downarrow$ & $0,0=$ & $-13,6 \downarrow$ & $-9,1 \downarrow$ & $27,3 \uparrow$ \\
\hline & Colaboración & $0,0=$ & $0,0=$ & $-36,4 \downarrow \downarrow$ & $4,5 \uparrow$ & $31,8 \uparrow$ \\
\hline & Reflexión & $-22,8 \downarrow \downarrow$ & $0,0=$ & $-4,5 \downarrow$ & $9,1 \uparrow$ & $22,7 \uparrow$ \\
\hline & Formación & $-22,8 \downarrow \downarrow$ & $-22,7 \downarrow \downarrow$ & $31,8 \uparrow \uparrow$ & $41,0 \uparrow$ & $9,1 \uparrow$ \\
\hline & Búsqueda & $0,0=$ & $-27,3 \downarrow \downarrow$ & $-9,1 \downarrow$ & $27,3 \uparrow$ & $9,1 \uparrow$ \\
\hline & $\begin{array}{l}\text { Creación y } \\
\text { modificación }\end{array}$ & $-18,2 \downarrow$ & $4,5 \uparrow$ & $-45,5 \downarrow \downarrow$ & $\begin{array}{r}40,9 \uparrow \\
\text { 个 }\end{array}$ & $18,2 \uparrow$ \\
\hline & Protección & $-9,1 \downarrow$ & $0,0=$ & $-18,2 \downarrow$ & $13,6 \uparrow$ & $13,6 \uparrow$ \\
\hline
\end{tabular}

Tal y como se muestra en la tabla y, tras la aplicación del CIMA, se ha producido un notable incremento de alumnado que se sitúa en los niveles más altos (4 y 5). Al mismo tiempo, el número de alumnos y alumnas que se situaban en los niveles más bajos (1,2 y 3) ha disminuido. También se observan determinados niveles en los que el número de alumnos y alumnas sigue siendo el mismo. Este fenómeno ocurre, sobre todo, en el nivel 2: alumnado que sigue sin recordar cómo se resuelven problemas básicos con TIC.

\section{Conclusiones}

Me ha quedado claro que el trabajo grupal es muy beneficioso para el alumnado, si se sabe manejar adecuadamente. Además, es esencial incorporar preguntas inspiradoras o conflictos cognitivos en los que se hace dudar al alumnado. Éste debe expresar sus ideas libremente, siempre con una guía de la actividad, supervisión personalizada del docente y posterior entrega de un informe que deje constancia del aprendizaje desarrollado. Por este motivo, trataré de incorporar a mis futuras clases distintas "preguntas inspiradoras" o conflictos cognitivos a los que el alumnado no está acostumbrado; entrega de un guion de la actividad donde se deje claro qué se espera de ellos y ellas; actividades de trabajo en pequeños grupos; 
presencia activa del profesor; dar consejos personalizados que ayuden a resolver los problemas funcionales y cognitivos del grupo; entrega de un informe final.... A parte de todo esto, hay algo que me ha llamado enormemente la atención en el desarrollo del CIMA y que aprendí en una de las sesiones de formación, cuando analizábamos uno de los capítulos de la obra de Finkel (2008). A la hora de resolver los problemas del grupo, el docente no debe quedarse de pie, sino que debe sentarse con el grupo. Este simple gesto dice mucho de su forma de concebir la educación. Al sentarte con el grupo, te conviertes en un miembro más, rompiendo con la tradicional relación de poder del docente sobre el alumno. A partir de ahora, trataré de llevarlo a cabo en las clases.

En conclusión, la aplicación de este CIMA ha servido para tener mayor confianza en el alumnado y en mí mismo. El rechazo del poder tradicional del profesor, otorgando mayor protagonismo a las alumnas y alumnos, hace que las clases se conviertan en un espacio para explorar y encontrar; dudar, debatir y reflexionar; errar y mejorar. Aquel donde experimentan y aprenden personas distintas.

Jornadas de Formación e Innovación Docente del Profesorado | № 2 (2019) Esta obra se distribuye con la licencia Creative Commons 


\section{Referencias}

Bain, K. (2005). Lo que hacen los mejores profesores universitarios. Valencia: Universitat de Valencia.

Consejo de la UE. (2018). Recomendación del Consejo, de 22 de mayo de 2018, relativa a las competencias clave para el aprendizaje permanente. Bruselas: Diario Oficial de la UE.

Finkel, D. (2008). Dar clase con la boca cerrada. Valencia: Universitat de València.

Redecker, C., y Punie, Y. (2017). Digital Competence of Educators DigCompEdu. Luxemburgo: Servicio de publicaciones de la UE.

Rivero, A. y Porlán, R. (2017). La evaluación en la enseñanza universitaria. En Porlán, R. (Coord.), Enseñanza Universitaria. Cómo mejorarla (pp. 73-91). Madrid: Ediciones Morata.

Jornadas de Formación e Innovación Docente del Profesorado | № 2 (2019) Esta obra se distribuye con la licencia Creative Commons 